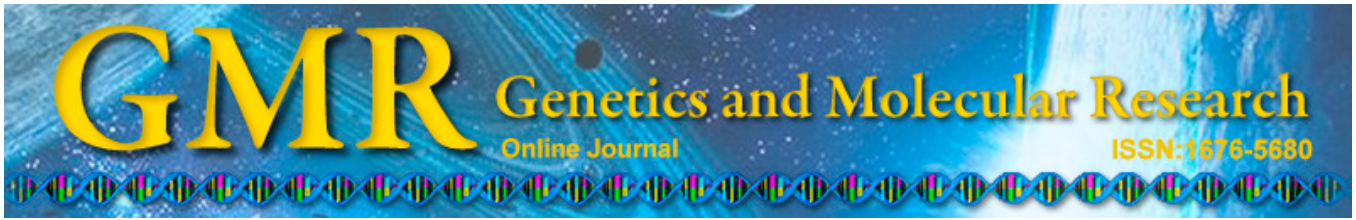

\title{
Involvement of the mitfa gene in the development of pigment cell in Japanese ornamental (Koi) carp (Cyprinus carpio L.)
}

J.H. Liu ${ }^{1 *}$, S. Wen ${ }^{1 *}$, C. Luo ${ }^{1}$, Y.Q. Zhang ${ }^{1}$, M. Tao ${ }^{1}$, D.W. Wang ${ }^{2}$, S.M. Deng ${ }^{2}$ and Y.M. Xiao ${ }^{1}$

${ }^{1}$ Key Lab of Protein Chemistry and Developmental Biology of Education, Ministry of China, College of Life Sciences, Hunan Normal University, Changsha, China

${ }^{2}$ Hunan Fisheries Research Institute, Changsha, China

*These authors contributed equally to this study.

Corresponding author: Y.M. Xiao

E-mail: yameix@126.com

Genet. Mol. Res. 14 (1): 2775-2784 (2015)

Received February 25, 2014

Accepted August 18, 2014

Published March 31, 2015

DOI http://dx.doi.org/10.4238/2015.March.31.7

ABSTRACT. A colored phenotype is an important feature of ornamental fish. In mammals, microphthalmia-associated transcription factor (MITF) was found to regulate the development of melanocytes. In this study, the mitfa cDNA was first cloned from the Japanese ornamental (Koi) carp (Cyprinus carpio L.), an important ornamental freshwater fish. The full-length cDNA of the mitfa gene contains $1634 \mathrm{bp}$, coding for 412 amino acids in Koi. The identity degree of mitfa amino acid sequences between the Koi carp and zebrafish is $92.9 \%$. We tested the expression of the mitfa gene in several varieties of Koi using reverse transcription-polymerase chain reaction and found that the mitfa gene is highly expressed in the skin tissues of the Taisho sanke and the Procypris merus. Interestingly, the mitfa gene was also expressed in the Kohaku and Yamabaki ogon, although melanocytes were not observed in the skin. Koi carp embryos were transparent and colorless, while 
after hatching, different types of pigment cells successively emerged in a fixed order. In Taisho sanke, melanocytes first appeared in the trunk at approximately 12 days of age. Subsequently, there was a large area of melanocytes by 30 days of age. The expression level of the mitfa mRNA was low in early embryos and newly hatched larvae, and increased to high levels in 30-day-old fry. The results show that the mitfa gene is involved in regulating fish body color in the development of both melanocytes and pigment cells.

Key words: Japanese ornamental (Koi) carp; Melanocyte; mitf gene; Pigment cell

\section{INTRODUCTION}

Color patterns are prominent features of many animals and play important roles in camouflage, shoaling, mate choice, the perception of threatening behavior, or the protection against UV irradiation (Protas and Patel, 2008; Howe et al., 2013; Lin et al., 2013). Pigment phenotypes in fish are more complicated than in amniota. There are 4 types of pigment cells in fish, including melanocytes, erythrocytes, xanthophores, and iridocytes (Kelsh, 2004). Various factors are thought to be involved in regulating these cells, such as Fms, Pax3, Sox10, and Wnt (Parichy and Turner, 2003; Lang and Epstein, 2003; Minchin and Hughes, 2008; Koludrovic and Davidson, 2013). The microphthalmia-associated transcription factor (MITF) is a member of the basic helix-loop-helix leucine zipper (bHLH-Zip) protein family (Hodgkinson et al., 1993). The mitf gene is expressed in most cell types, and it plays major roles in neural crest-derived and neuroepithelium-derived pigment cells. In mammals, the mitf gene direction regulates the differentiation of melanin cell by controlling the expression of tyrosine gene families (Odenthal et al., 1996; Levy et al., 2006). Mutations in the mitf gene cause pigmentation diseases, such as deafness, dystopia canthorum, or melanomas (Tassabehji et al., 1994; Smith et al., 2000; Levy et al., 2010; Yokoyama, et al., 2011).

In humans, the mitf gene is located on the chromosome $3 \mathrm{P}^{14.1}-3 \mathrm{P}^{12.3}$ (Garraway et al., 2005). Because of the different promoters used, diverse transcriptional regulation occurs for the mitf gene, resulting in the production of multiple MITF isoforms from alternative transcription or splicing (Tachibana et al., 1996; Amae et al., 1998; Fuse et al., 1999). The mitf gene in human produces 18 transcripts, 14 of which can be translated into proteins; there are 9 named subtypes and additional unnamed subtypes. MitfA, MitfD, and MitfH are necessary for retinal pigment epithelium development, while MitfM plays a critical role in the development of melanocytes in humans (Oboki et al., 2002; Li et al., 2013; Koludrovic and Davidson, 2013). The mitfa and mitfb genes have also been reported, which are located on chromosomes 6 and 23 in zebrafish. Additionally, the mitfa gene is closely related to the development of melanocytes, while the mitfb gene is involved in the retinal pigment epithelium development in zebrafish (Curran et al., 2010; Li et al., 2014).

The Koi (Cyprinus carpio) carp is a variety of common carp and an important ornamental fish in Asia. In this study, we cloned the mitfa cDNA from Koi carp. We analyzed the expression of the mitfa gene in varieties of Koi carp with different body colors and at different stages of body color development. Our results are useful for determining the molecular mechanism of pigment development in the ornamental fish body. 


\section{MATERIAL AND METHODS}

\section{Materials}

Koi carp (6 months old) were obtained from the Hunan YuYuan biological Technology Co., Ltd. in Changsha, Hunan, China. The fertilized eggs of the Koi carp were raised and hatched in the laboratory.

The tissues of skin, scales, or fins of the Koi carp were quickly dissected and then soaked in phosphate buffer solution for directly observation under a microscope. The skin or embryos of Koi carp were collected and frozen in liquid nitrogen for total RNA extraction.

\section{Molecular cloning of full-length mitfa gene}

The full-length mitfa cDNA was cloned from Koi carp according to the mitfa cDNA sequence of zebrafish (GenBank accession No. NM130923). Total RNA was extracted from the skin of Koi carp (Procypris merus). Reverse transcriptionpolymerase chain reaction (RT-PCR) was conducted using $1 \mu \mathrm{L} 10 \mu \mathrm{M}$ mitfa $1^{+/}$primers (Table 1). PCR was conducted in a total volume of $20 \mu \mathrm{L}$ and run for 35 cycles. The rapid amplification of cDNA 3'-ends (RACE) was performed using Clontech Universal Primer A Mix solution (Mountain View, CA, USA) (longer primer: $0.4 \mu \mathrm{M}$ 5'-CTAATACGACTCACTATAGGGCAAGCAGTGGTATCAACGCAGAGT-3', shorter primer: $2 \mu \mathrm{M}$ 5'-CTAATACGACTCACTATAGGGC-3'), and mitfa3-out primer (Table 1), for 30 cycles. PCR products were re-amplified using $1 \mu \mathrm{L} 10 \mu \mathrm{M}$ mitfa3-in primer (Table 1) and Nested Universal Primer A (5'-AAGCAGTGGTATCAACGCAGAGT-3'). 5'-RACE was performed using the 5-RACE kit purchased from Clontech Laboratories, Inc. The PCR procedures were the same as those described above, using mitfa $5^{+/}$primers (Table 1 ) and Universal Primer A Mix solution. Amplified products were gel-purified and cloned into the PMD18-T vector (Takara, Shiga, Japan) for sequencing (Xiao et al., 2014).

Table 1. Oligo primers used for mitfa cDNA cloning or RT-PCR analysis from the Koi carp.

\begin{tabular}{ll}
\hline Primer name & Primer sequences $\left(5^{\prime}-3^{\prime}\right)$ \\
\hline$\beta$-actin+ & CCGTGACCTGACTACCCTC \\
$\beta$-actin- & ATACCGCAAGATTCCATACCC \\
mitfa-1+ & ACAACTCCTGCCCGTCTAAC \\
mitfa-1- & CCGTTGTTGAGTCCAGAGT \\
mitfa+ & CTACAGTGATGACATTCTTGGGTTC \\
mitfa- & CCTTGTTTGGGCTGTCGTAG \\
mitfa3-out & GAAACTCCAGAAAGAGCAGCAA \\
mitfa3-in & AGCCTCACCCAGCCTTTATT \\
mitfa5-out & CTTTTACCAAGGCTCTGACCTCTGC \\
mitfa5-in & CGGTAGGTTAGACGGGCAGGAGTTGTT \\
NUP & AAGCAGTGGTATCAACGCAGAGT \\
3 '-primer & AAGCAGTGGTATCAACGCAGAGTACT ${ }_{16}$ VN \\
UPM $_{\text {(shor) }}$ & CTAATACGACTCACTATAGGGC \\
PPM $_{\text {(long) }}$ & CTAATACGACTCACTATAGGGCAAGCAGTGGTATCAACGCAGAGT \\
\hline
\end{tabular}

\section{RT-PCR}

Reverse transcription was conducted in a total reaction volume of $20 \mu \mathrm{L}$, which in- 
cluded $2 \mu \mathrm{g}$ total RNA and $2 \mu \mathrm{L}$ RT reaction mixture. For PCR amplification, both specific primers and $\beta$-actin primers were added into the same reaction at the beginning of PCR and each PCR run for 30 cycles. The PCR products were separated by agarose gel electrophoresis and photographed under UV illumination. We list the primers used in the experiments in Table 1. For each batch, the experiment was repeated more than 3 times.

\section{RESULTS}

\section{Molecular cloning and sequence analysis of the mitfa gene from the Koi carp}

The full-length mitfa cDNA was cloned from the Koi carp (GenBank accession No. KC565527). The mitfa cDNA gene from the Koi carp was 1634 bp long and contained an open reading frame of $1236 \mathrm{bp}$, coding for 412 amino acids (Figure 1).

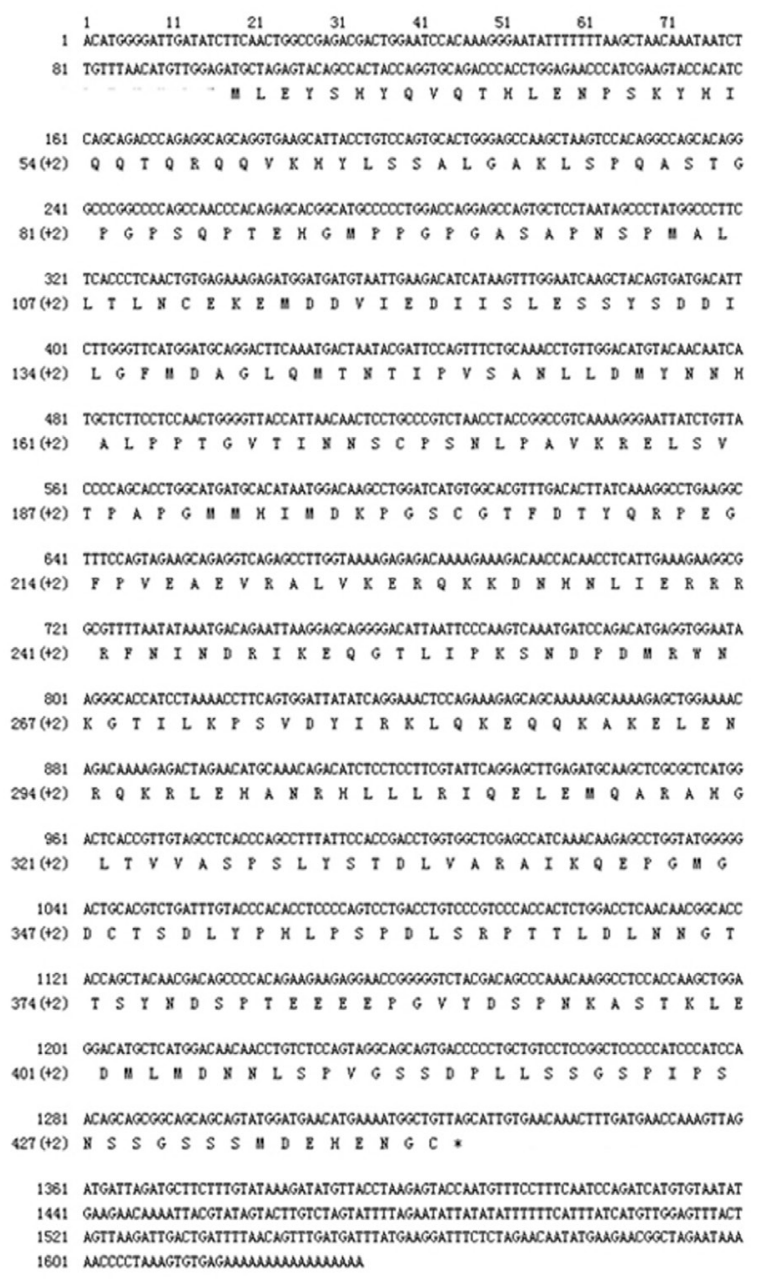

Figure 1. Multiple nucleotide sequence and amino acid sequence alignment of mitfa in Koi carp. 
We compared the cDNA sequences of mitfa from Koi carp and 5 other fish, including Amphilophus citrinellus (GenBank accession No. AY206404), Astatotilapia burtoni (GenBank accession No. AY206400), Maylandia zebra (GenBank accession No. AY196318), zebrafish (GenBank accession No. NM130923), and Paralichthys olivaceu (GenBank accession No. AB457038) (Figure 2). This analysis revealed a high level of sequence similarity in the mitfa gene among these fish. The mitfa nucleotide and amino acid sequences from the Koi carp and zebrafish were 84 and $92.9 \%$ identical, respectively.

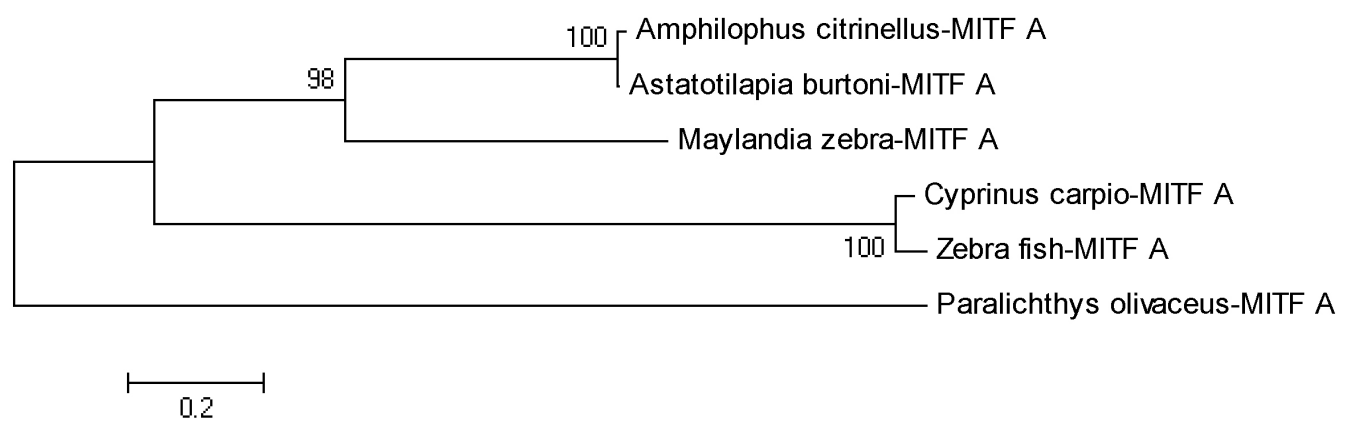

Figure 2. Phylogenetic tree of the mitfa gene in the 6 species (Amphilophus citrinellus; Astatotilapia burtoni; Maylandia zebra; Cyprinus carpio; zebrafish; Paralichthys olivaceus) from NCBI. This phylogenetic tree was generated by comparative analysis of the mitfa coding sequences and using unweighted pair group method with arithmetic mean calculation and the MEGA5 software.

\section{Expression of the mitfa gene in skin of the Koi carp}

The body color of P. merus was black or has white spots (Figure 3A). There were 3 types of the pigment cells present, including melanophores, erythrophores, and xanthophores, in the skin tissue, scales, or fins of P. merus (Figure 3B-D). The body color of Taisho sanke was white with red and black spots (Figure $3 \mathrm{E}$ ) and contained melanophores, erythropores, and lipophores in the skin, scales, and fins (Figure 3F-H). Kohaku showed some red spots on their white bodies (Figure 3I), consisting of erythropores and xanthophores, while melanophores were not observed (Figure 3J-L). The whole body color Yamabaki ogon was yellow (Figure $3 \mathrm{M}$ ), with an abundance of erythropores and xanthophores in the skin, scales, and fins (Figure $3 \mathrm{~N}-\mathrm{P})$. Iridocytes, 1 type of pigment cells, was observed in the skin and scales of the Koi carp.

We conducted RT-PCR to explore whether the mitfa gene is differentially expressed in different varieties of Koi carp. The result (Figure 4) showed that higher levels of the mitfa mRNA are expressed in the skin of $P$. merus and T. sanke. Additionally, mitfa was expressed in the skin of Kohaku and Y. ogon, although mitfa mRNA levels were lower than in P. merus and T. sanke.

\section{Expression of the mitfa gene during body color development in Koi carp}

T. sanke, which has 4 types of pigment cells, was examined for body color change during development. The embryos of $T$. sanke were transparent, and no pigment cells were observed in its body except in the eyes. Xanthophores were observed on the first day after hatching. The number of xanthophores increased and gradually appeared from the head to tail in the fry of $T$. sanke. Iridocytes emerged on the belly after 7 days. Melanophores were first 
observed after 12 days, and their number had increased by 24 days, when they were widely distributed throughout the body (Figure 5).

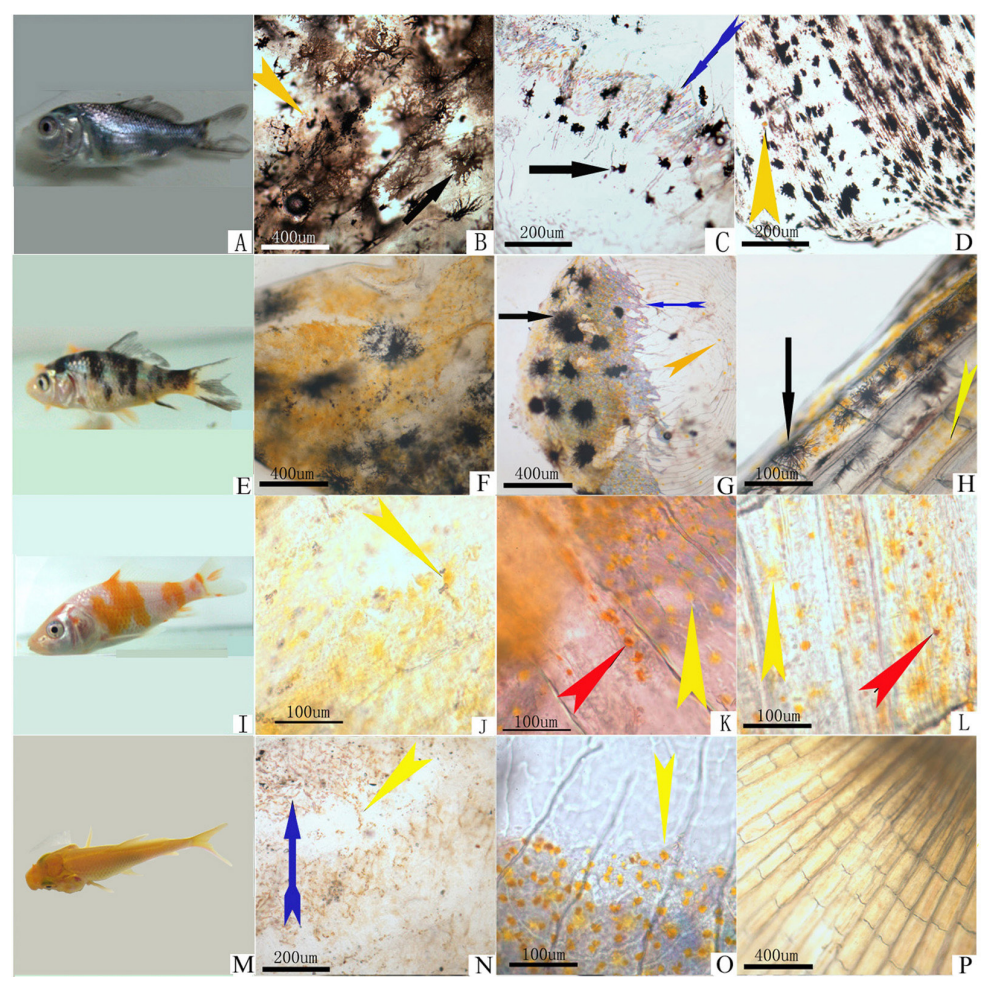

Figure 3. Four varieties of Koi carp and microscopic observation of their pigments. A.-D. Procypris merus. E.-H. Taisho sanke. I.-L. Kohaku. M.-P. Yamabaki ogon. The photos from the second to the fourth columns are derived from the tissues of the skin, scale, and fin, respectively. Simple arrow indicates the melanocyte; gray arrowhead indicates the xathophore; black arrowhead indicates the erythrophore; bifurcated tail end arrow indicates the iridocyte.
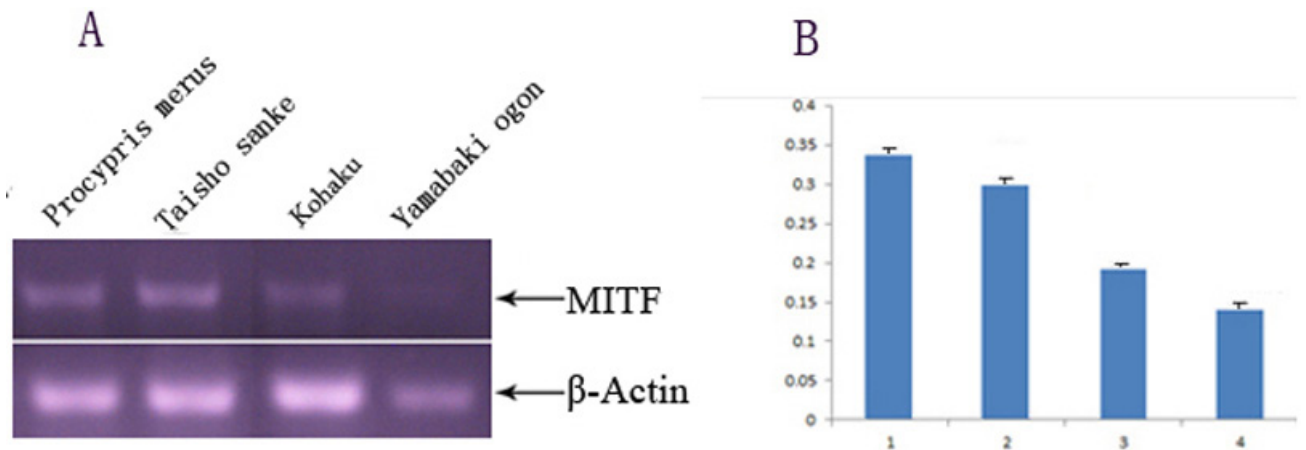

Figure 4. RT-PCR analysis revealed levels of the mitfa mRNA among of Koi carp, including Procypris meru, Taisho sanke, Kohuku, and Yamabaki ogon (A). The relative level of expression was calculated by dividing the total pixel from each mitfa mRNA band with the total pixel from the corresponding $\beta$-actin mRNA band. Column 1 indicates P. merus; 2 indicates T. sanke; 3 indicates Kohaku; 4 indicates Y. ogon (B). Quantitative results are from 3 independent experiments. 


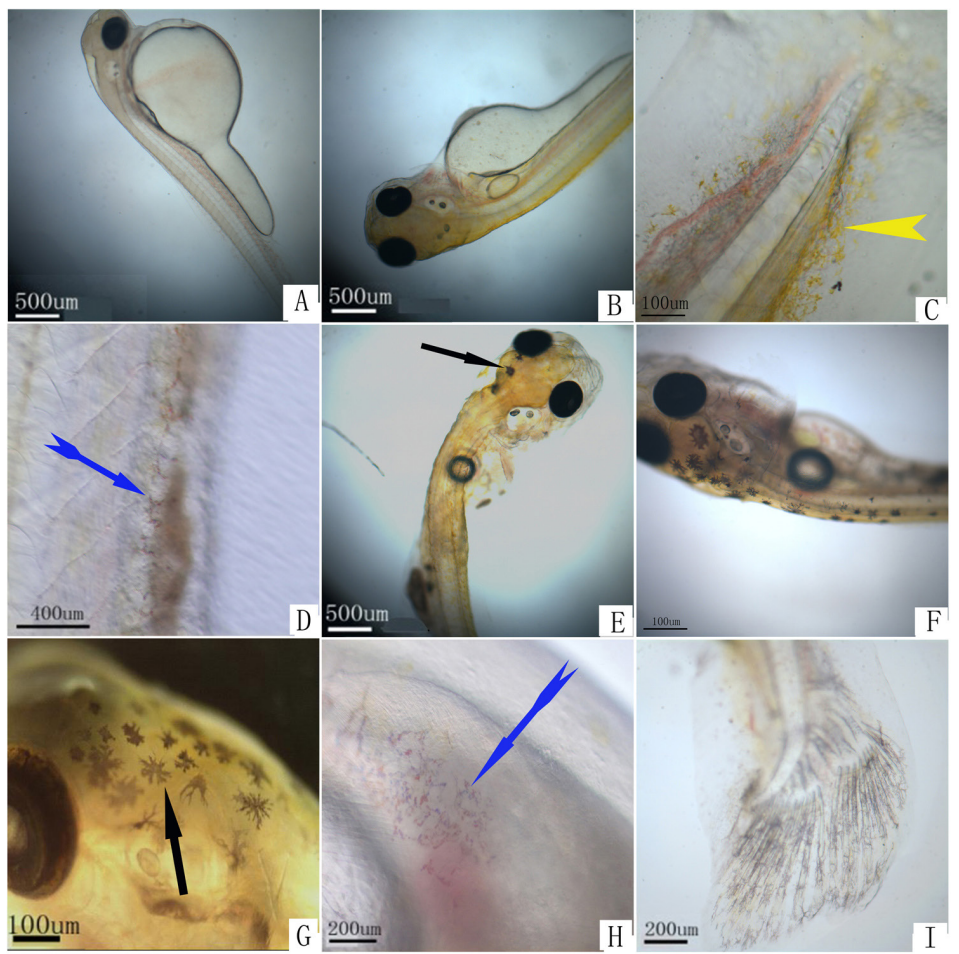

Figure 5. Early development of body pigment in Taisho sanke. A. 1 dph. B. and C. 3 dph. D. 7 dph. E. 10 dph. F. 14 dph. G. 16 dph. H. 18 dph. I. 24 dph. Gray arrowhead indicates the xathophore; black arrowhead indicates the erythrophore; bifurcated tail end arrow indicates the iridocyte, simple arrow shows the melanocyte. $\mathrm{dph}=$ days post-hatching.

Total RNA was extracted from the blastula, 1-day-old larvae, and 30-day-old fry of T. sanke. RT-PCR analysis showed that the level of the mitfa mRNA was lower at the blastula stage and in 1-day-old larvae of T. sanke, with high expression of the mitfa mRNA in 30-dayold T. sanke.

\section{DISCUSSION}

In mammals, MITF consists of 413-520 amino acids and is a member of the basic helix-loop-helix leucine zipper (bHLH-Zip) protein family (Pogenberg et al., 2012). In this study, we cloned the mitfa cDNA from Koi carp, which shows high homology in the nucleotide sequences of Koi carp and zebrafish. Based on our analysis of the amino acid sequence of MITFa in Koi carp using Smartprotein, we found 3 domains: the amino acids from 61-76 constitute the low abundance phenotype, the bHLH-Zip is formed by amino acids 205-258, and 285-409 constitutes an domain (DUF3371), which contains a set of bHLH transcription factors, the members in the family of MIT/TFE (MITF transcription factor). Analysis with EXPASY revealed that amino acids 199-252 form Myc bHLH-Zip in Koi carp. Compared with mammals, there are 2 amino acid mutations at sites of 222 and 244 in the bHLH of MITFa in zebrafish and Koi carp. 
MITF is a biomarker of differentiation from neural crest cells to melanin in mammals (Goding, 2000). It can regulate the expression of tyrosinase, which is an enzyme important in melanin synthesis (Hodgkinson et al., 1993; Lister et al., 2001). The zebrafish MITFb is mainly expressed in retinal pigment epithelium, and its ectopic expression can rescue the pigment phenotype of the mitfa mutant (Lister et al., 1999). Therefore, in zebrafish, the 2 mitf genes have undergone subfunctionalization to provide complementary expression and functions in pigmentation (Lister et al., 2001; Altschmied et al., 2002). In this study, we examined whether in Koi carp with different body colors, or in the ontogenesis of Koi carp, expression of the mitfa gene was closely associated with melanin abundance in the cell. Notably, expression of the mitfa gene was also detected in Koi carp without melanocytes in the skin, including Kohaku, Y. ogon, and Koi embryos or hatched larvae.

Pigment cells are derived from the embryonic neural crest (Hultman and Johnson, 2010). Skin pigmentation is a complex process involving a series of cellular, genetic, and physiological factors (Colihueque, 2010). In response to external stimuli, multiple signals are generated by non-melanocytic cells in the epidermis, which communicate a pigmentation response to the basal melanocyte population. Alpha-melanocyte-stimulating hormone is one such peptide hormone that has been suggested to modulate the pigment response (Thody, 1999). Although cAMP triggers numerous downstream effects, an important target is the MITF gene, which is transcriptionally upregulated by cAMP signaling in a melanocyte-restricted fashion, thus linking extracellular signals to MITF expression and the transcriptional regulation of pigmentation (Park et al., 2006). Warrdenburg syndrome is caused by mitf gene mutations in human, which can result in skin pigment loss and the production of abnormal ididocytes (Pingault et al., 2010). Both melanocytes and the iris are thought to be derived from the same precursor cells. In the zebrafish containing the mitf gene mutant, the iris was found to be overexpressed, and the black patches disappeared (Rawls et al., 2001), whereas a reduction in melanophore number and stripe formation was caused by the absence of iridophores (Howe et al., 2013). Pnp4a is considered a marker of iridoblasts and co-regulates the differentiation and development of melanin cell lineage and iridescent cell lineage together with MITF and foxd3 (Curran et al., 2010). Curran et al. (2010) suggested that melanoblasts and iridoblasts originate from the same precursor cells. However, $\mathrm{Tu}$ and Johnson (2010) found that melanocytes and xanthophores originate from the same precursor stem cell, while iridocytes were produced by other cells in zebrafish. We found that the mitfa gene was involved in the regulation of fish body color, not only in melanocytes, but also in other pigment cells. Skin color determination is a very complex process in fish associated with a series of cellular, genetic, environmental, and physiological factors (Aspengren et al., 2009). Additional studies are needed to determine the strategy of pigment cell development in fish.

\section{ACKNOWLEDGMENTS}

Research supported by Hunan Provincial Natural Science Foundation ofChina (\#14JJ2058), the National Science Foundation of China (\#31472272 to Y.M. Xiao), the Hunan Provincial Science and Technology Program (\#2014NK3039 to J.H. Liu), and the Cooperative Innovation Center of Engineering and New Products for Developmental Biology of Hunan Province.

\section{REFERENCES}

Altschmied J, Delfgaauw J, Wilde B, Duschl J, et al. (2002). Subfunctionalization of duplicate mitf genes associated with differential degeneration of alternative exons in fish. Genetics 16: 259-267. 
Amae S, Fuse N, Yasumoto K, Sato S, et al. (1998). Identification of a novel isoform of microphthalmia-associated transcription factor that is enriched in retinal pigment epithelium. Biochem. Biophys. Res. Commun. 247: 710-715.

Aspengren S, Sköld HN and Wallin M (2009). Different strategies for color change. Cell. Mol. Life Sci. 66: 187-191.

Colihueque N (2010). Genetics of salmonid skin pigmentation: clues and prospects for improving the external appearance of farmed salmonids. Rev. Fish Biol. Fish. 20: 71-86.

Curran K, Lister JA, Kunkel GR, Prendergast A, et al. (2010). Interplay between Foxd3 and Mitf regulates cell fate plasticity in the zebrafish neural crest. Dev. Biol. 344: 107-118.

Fuse N, Yasumoto K, Takeda K, Amae S, et al. (1999). Molecular cloning of cDNA encoding a novel microphthalmiaassociated transcription factor isoform with a distinct amino-terminus. J. Biochem. 126: 1043-1051.

Garraway LA, Widlund HR, Rubin MA, Getz G, et al. (2005). Integrative genomic analyses identify MITF as a lineage survival oncogene amplified in malignant melanoma. Nature 436: 117-122.

Goding CR (2000). Mitf from neural crest to melanoma: signal transduction and transcription in the melanocyte lineage. Genes Dev. 14: 1712-1728.

Hodgkinson CA, Moore KJ, Nakayama A, Steingrimsson E, et al. (1993). Mutations at the mouse microphthalmia locus are associated with defects in a gene encoding a novel basic-helix-loop-helix-zipper protein. Cell 74: 395-404.

Howe K, Clark MD, Torroja CF, Torrance J, et al. (2013). The zebrafish reference genome sequence and its relationship to the human genome. Nature 496: 498-503.

Hultman KA and Johnson SL (2010). Differential contribution of direct developing and stem cell-derived melanocytes to the zebrafish larval pigment pattern. Dev. Biol. 337: 425-431.

Koludrovic D and Davidson I (2013). MITF, the Janus transcription factor of melanoma. Future Oncol. 9: 235-244.

Kelsh RN (2004). Genetics and evolution of pigment patterns in fish. Pigment Cell Res. 17: 326-336.

Lang D and Epstein JA (2003). Sox10 and Pax3 physically interact to mediate activation of a conserved c-RET enhancer. Hum. Mol. Genet. 12: 937-945.

Levy C, Khaled M and Fisher DE (2006). MITF: master regulator of melanocyte development and melanoma oncogene. Trends Mol. Med. 12: 406-414.

Levy C, Khaled M, Robinson KC, Vequilla RA, et al. (2010). Lineage-specific transcriptional regulation of DICER by MITF in melanocytes. Cell 141: 994-1005.

Li M, Zhu F and Hong Y (2013). Differential evolution of duplicated medakafish mitf genes. Int. J. Biol. Sci. 9: 496-508.

Li M, Zhu F, Hong N, Zhang L, et al. (2014). Alternative transcription generates multiple Mitf isoforms with different expression patterns and activities in medaka. Pigment Cell Melanoma Res. 27: 48-58.

Lin SJ, Foley J, Jiang TX, Yeh CY, et al. (2013). Topology of feather melanocyte progenitor niche allows complex pigment patterns to emerge. Science 340: 1442-1445.

Lister JA, Robertson CP, Lepage T, Johnson SL, et al. (1999). Nacre encodes a zebrafish microphthalmia-related protein that regulates neural-crest-derived pigment cell fate. Development 126: 3757-3767.

Lister JA, Close J and Raible DW (2001). Duplicate mitf genes in zebrafish: complementary expression and conservation of melanogenic potential. Dev. Biol. 237: 333-344.

Minchin JEN and Hughes SM (2008). Sequential actions of Pax3 and Pax7 drive xanthophore development in zebrafish neural crest. Dev. Biol. 317: 508-522.

Oboki K, Morii E, Kataoka TR, Jippo T, et al. (2002). Isoforms of mi transcription factor preferentially expressed in cultured mast cells of mice. Biochem. Biophys. Res. Commun. 290: 1250-1254.

Odenthal J, Rossnagel K, Haffter P, Kelsh RN, et al. (1996). Mutations affecting xanthophore pigmentation in the zebrafish, Danio rerio. Development 123: 391-398.

Parichy DM and Turner JM (2003). Temporal and cellular requirements for Fms signaling during zebrafish adult pigment pattern development. Development 130: 817-833

Park H, Wu C, Yonemoto L, Murphy-Smith M, et al. (2006). MITF mediates cAMP-induced protein kinase C-beta expression in human melanocytes. Biochem. J. 395: 571-578.

Pingault V, Ente D, Dastot-Le Moal F, Goossens M, et al. (2010). Review and update of mutations causing Waardenburg syndrome. Hum. Mutat. 31: 391-406.

Pogenberg V, Ögmundsdóttir MH, Bergsteinsdóttir K, Schepsky A, et al. (2012). Restricted leucine zipper dimerization and specificity of DNA recognition of the melanocyte master regulator MITF. Genes Dev. 26: 2647-2658.

Protas ME and Patel NH (2008). Evolution of coloration patterns. Annu. Rev. Cell Dev. Biol. 24: 425-446.

Rawls JF, Mellgren EM and Johnson SL (2001). How the zebrafish gets its stripes. Dev. Biol. 240: 301-314.

Smith SD, Kelley PM, Kenyon JB and Hoover D (2000). Tietz syndrome (hypopigmentation/deafness) caused by mutation of MITF. J. Med. Genet. 37: 446-448.

Tachibana M, Takeda K, Nobukuni Y, Urabe K, et al. (1996). Ectopic expression of MITF, a gene for Waardenburg 
syndrome type 2, converts fibroblasts to cells with melanocyte characteristics. Nat. Genet. 14: 50-54.

Tassabehji M, Newton VE and Read AP (1994). Waardenburg syndrome type 2 caused by mutations in the human microphthalmia (MITF) gene. Nat. Genet. 8: 251-255.

Thody AJ (1999). AlphaMSH and the regulation of melanocyte function. Ann. N Y Acad. Sci. 885: 217-229.

Tu S and Johnson SL (2010). Clonal analyses reveal roles of organ founding stem cells, melanocyte stem cells and melanoblasts in establishment, growth and regeneration of the adult zebrafish fin. Development 137: 3931-3939.

Xiao YM, Jiang MG, Luo ZW, Zhou YH, et al. (2014). Identification and analysis of the jnk1 gene in polyploid hybrids of red crucian carp (Carassius auratus red var.) and common carp (Cyprinus carpio L.). Genet. Mol. Res. 13: 906-919.

Yokoyama S, Woods SL, Boyle GM, Aoude LG, et al. (2011). A novel recurrent mutation in MITF predisposes to familial and sporadic melanoma. Nature 480: 99-103. 